\title{
CSCW e GROUPWARE: contexto no desenvolvimento de software em ambiente fisicamente distribuído
}

\author{
Roberto Zanoni ${ }^{1}$ \\ ${ }^{1}$ Faculdade de Tecnologia SENAC Passo Fundo/RS - Coordenador do Curso Superior \\ de Tecnologia em Sistemas de Informação. \\ robzanoni@yahoo.com.br/robzanoni@gmail.com
}

\begin{abstract}
Resumo. O objetivo deste trabalho é contextualizar CSCW e Groupware no Desenvolvimento de Software em Ambiente Fisicamente Distribuído, onde os atores se encontram distantes fisicamente, aflorando vários problemas relacionados à falta de trabalho colaborativo. Destacam-se dois fatores de grande influência na causa desses problemas, as diferenças culturais e o idioma. A pesquisa caracteriza-se como um estudo qualitativo, adequado quando se objetiva o desenvolvimento de novos métodos e modelos. Os resultados apontam para a necessidade do desenvolvimento de tecnologias que auxiliem a atuação de pessoas que estão inseridas em ambientes distantes fisicamente e que queiram trabalhar de forma colaborativa.
\end{abstract}

\section{Introdução}

O principal objetivo das aplicações colaborativas é proporcionar aos usuários ferramentas que lhes permitam coordenar suas atividades de trabalho. Apesar de haver uma diversidade de conceitos de trabalho cooperativo, assim como distintas aplicações, todos têm um fator em comum que consiste em tornar mais fácil o processo de compartilhar informações entre pessoas que realizam uma ou várias tarefas em comum. Com o intuito de apoiar tecnologicamente grupos de trabalhos cooperativos, provendoos de uma ferramenta e serviços através de uma interface em um ambiente distribuído, que surge a tecnologia de groupware. Os sistemas considerados de groupware se apóiam dentro da área de investigação do CSCW - Trabalho Cooperativo Suportado por Computador - que pode ser visto como uma disciplina que guia a correta análise, projeto e desenvolvimento de sistemas colaborativos (BOURGES, 1999).

A globalização da economia tem levado muitas organizações a distribuírem geograficamente seus recursos e investimentos visando obter melhores resultados. Ambiente fisicamente distribuído é uma nova tendência de desenvolvimento de software, onde usuários e equipe de desenvolvimento estão em locais físicos diferentes, às vezes países, com culturas diferentes. A área de desenvolvimento de software foi pioneira neste processo de distribuição, em paises como Índia e Irlanda. Maior produtividade, redução de custos, minimização dos riscos e qualidade, são algumas características que grandes organizações buscam com a distribuição de seus processos fora de seus países. Neste sentido, a globalização está aumentando a demanda de sistemas CSCW que suportem grupos de trabalho geograficamente distribuídos nos quais os usuários possam trabalhar em tempo real. A criação deste tipo de sistema representa novos desafios para os desenvolvedores. Um dos desafios mais importantes á 
o de evitar os problemas de usabilidade provocados pela diversidade de usuários de diferentes culturas.

O objetivo deste artigo é contextualizar CSCW e Groupware no Desenvolvimento de Software em Ambiente Fisicamente Distribuído, onde os atores se encontram distantes fisicamente, aflorando vários problemas relacionados à falta de trabalho colaborativo. A pesquisa caracteriza-se como um estudo qualitativo, adequado quando se tem por objetivo o desenvolvimento de novos métodos e modelos. Este artigo apresenta, na seção 2, uma base teórica referente a CSCW e Groupware, destacando os problemas relacionados com o idioma. Na seção 3, a Distribuição Física é relatada, juntamente com os seus problemas e um comparativo entre um ambiente centralizado e um ambiente distribuído. Na seção 4, é descrito um protótipo para auxílio do trabalho colaborativo voltado para ambiente fisicamente distribuído. Por fim, a conclusão é apresentada na seção 5 .

\section{CSCW e Groupware}

O CSCW inclui uma grande variedade de aplicações que vão desde editores cooperativos até sistemas de engenharia concorrente e de teleconferência. Estas aplicações favorecem a integração dos usuários e permitem um aumento do rendimento na execução das tarefas, em relação ao trabalho individual isolado. Além do mais, a contínua evolução tecnológica tem projetado o trabalho cooperativo no mundo das aplicações em redes geograficamente distribuídas, oferecendo a possibilidade de intercâmbio de idéias e de execução de tarefas em conjunto para um número maior de pessoas.

Numa visão prática, groupware é considerado qualquer ferramenta que ajuda as pessoas a trabalharem juntas mais fácil e eficazmente, citando o telefone como a mais simples e comumente utilizada dessas ferramentas. Para o contexto computacional, à groupware está associado o conceito de um conjunto de hardware e software numa rede, que procura atender três propósitos: 1 . Comunicação - Ajudar pessoas a compartilhar informações; 2. Coordenação - Ajudar pessoas a coordenar seus papéis pessoais com os outros; 3. Colaboração - Ajudar pessoas a trabalhar juntas (BOURGES, 1999).

Existem ferramentas para uso colaborativo. Em geral essas ferramentas são divididas em três classes: videoconferências (como por exemplo, o NetMeeting, o Webex e o Click to Meet), ambientes virtuais colaborativos (também conhecidos como CVEs - Collaborative Virtual Environments - exemplos: Active Worlds, The Palace, o Blaxxun e o DIVE) e o learningware (aprendizagem colaborativa na Web, como exemplo, podemos citar o AulaNet - www.aulanet.com.br). São ferramentas que suportam a Internet para poder suprir seus requisitos de groupware, pois a descentralização que é permitida desta forma respeita a maioria dos seus requisitos.

\subsection{CSCW e os Problemas com o Idioma}

Na literatura existente, os aspectos culturais dos sistemas CSCW normalmente se centram na identificação e classificação de problemas específicos de usabilidade causados por diferenças culturais. Em SCRIVENER et al. esses problemas são classificados em seis grandes áreas: Convenções sócio-lingísticas; Convenções tipográficas; Ícones e símbolos; Instituições; Convenções psico-sociais e; Idioma.

Segundo BOURGES, esta classificação é útil para reconhecer os problemas que 
acontecem quando usuários de diferentes culturas interagem através de sistemas CSCW. Porém, é importante que estes aspectos sejam considerados pelos desenvolvedores de sistemas para tentar minimizar os problemas que estas diferenças produzem.

A globalização tem impulsionado o aumento da demanda pelas aplicações CSCW, onde a cooperação nas empresas torna-se imperativa, principalmente porque muitas têm seus escritórios em diferentes partes do mundo. É igualmente inevitável que o trabalho cooperativo represente uma mudança de paradigma uma vez que modificamse as formas tradicionais de trabalho e o ambiente de comunicação organizacional.

Segundo EVARISTO, esta mudança de paradigma é resultante da convergência de uma série de fenômenos. Dentre os principais, podemos destacar: as oportunidades decorrentes da disponibilidade e ampliação de uso das redes de computadores; o desejo de atender a pequenos grupos de trabalhos estabelecidos (workgroups), simultaneamente com requintes de comunicação e interação; a execução de pesquisas e desenvolvimento de aplicações para prover suporte a decisão a grupos de executivos e gerentes; a conjunção de telecomunicações e computadores, eliminando limitações geográficas e proporcionando utilização de recursos e mídias de representação antes inimagináveis; a globalização dos mercados levando as organizações a novas formulações em termos de estruturas e parcerias e a redefinição do ambiente de negócios.

No entanto, apesar dos sistemas CSCW serem ferramentas eficazes para juntar idéias, desenvolver pesquisas e trabalhos em colaboração entre profissionais de diferentes cantos do mundo, juntando conhecimentos e experiências que podem contribuir de forma significativa ao desenvolvimento das nações, um dos principais problemas enfrentados por seus usuários encontra-se na barreira da língua. Por isso, pelo menos uma das partes deve estabelecer a comunicação utilizando uma segunda língua, o que pode prejudicar a dinâmica da interação assim como a exatidão das informações trocadas.

\section{Desenvolvimento de Software em Ambiente Fisicamente Distribuído}

Gerenciar grandes projetos de software tem se tornado uma tarefa cada vez mais complexa. Não apenas por causa do crescimento dos projetos, mas também porque as equipes de projeto vêm se distribuindo no tempo e no espaço, inseridas no conceito de globalização que a sociedade tem vivenciado nos últimos anos. Isto configura, então, o desenvolvimento distribuído de software, no qual algumas pessoas envolvidas nesse processo estão fisicamente distantes (LAYZELL, 2000).

As empresas buscam vantagens competitivas como custos, qualidade ou flexibilidade na área de desenvolvimento de sistemas, além de ganhos de produtividade e diluição de riscos (GRUNDY, 1998). Nesse caso, ao optar por instanciar um ambiente de desenvolvimento distante fisicamente da sua sede, a organização começa a encarar desafios de adaptação, diferenças culturais, planejamento, treinamento, entre outros.

Neste contexto, surge um novo problema, que envolve, principalmente, a distância física entre os participantes do processo. Dessa forma, os já tradicionais problemas inerentes ao processo de desenvolvimento, fortemente centrados nas fases de especificação de requisitos e análise de sistemas, ganham contornos mais críticos. A forma de resolver esses problemas está centrada na adoção de linguagens de 
especificação e processos de gerência mais formais e definidos. Modelos de verificação e certificação do nível de maturidade do processo de desenvolvimento de software têmse tornado cada vez mais importantes e úteis para as organizações contratantes terem um mínimo de garantia sobre a qualidade do processo utilizado pela organização ou laboratório de desenvolvimento de sistemas parceiros (ZANONI, 2002).

Com a distribuição dos atores envolvidos, é necessário organizar e assegurar a qualidade desta integração. Para isto, é importante existirem modelos com capacidade de descrição e ambientes de automação computacional para os mesmos, possibilitando o emprego desses tipos de descrição para o suporte computacional do fluxo de trabalho.

Ao longo deste artigo, foram encontrados diversos critérios que podem caracterizar a distribuição física. Os critérios encontrados são: distância física dos atores; distribuição da equipe de desenvolvimento e; diferenças culturais (EVARISTO, 2001).

\subsection{Problemas relacionados à distribuição física}

GRUNDY et al. apontam alguns problemas criados ou exacerbados por alterações ocasionadas pela distribuição física. É interessante notar que, muito embora se fale dos problemas, existe pouca discussão das vantagens que o processo de gerenciamento e desenvolvimento distribuído oferece. A principal, e óbvia, é a possibilidade de reunir em uma equipe um conjunto de desenvolvedores geograficamente dispersos.

Os problemas apontados por GRUNDY são: a atribuição das tarefas do desenvolvimento aos membros da equipe; a comunicação e colaboração entre desenvolvedores; o compartilhamento dos documentos e artefatos do projeto, e a consistência destes; a necessidade de suportar plataformas heterogêneas; o acompanhamento do progresso dos grupos distintos; a integração entre os módulos desenvolvidos por estes grupos. Esses problemas são tratados em diversos trabalhos da área (FIELDING, 1996; HERBSLEB, 1999), e algumas soluções são discutidas.

\subsection{Ambiente centralizado $\mathrm{x}$ ambiente distribuído}

Vários aspectos diferenciam a atuação em um ambiente centralizado de um ambiente distribuído. A caracterização de um ambiente distribuído ocorre quando pelo menos um dos atores envolvidos (equipe de desenvolvimento, clientes, usuários) estiver fisicamente distante dos demais. A gerência de projeto, em um ambiente distribuído, deve abordar, de forma diferenciada, algumas características consideradas fatores de sucesso nesse tipo de ambiente, conforme tabela 1.

Tabela 1 Fatores de sucesso na gerência em ambiente fisicamente distribuído 


\begin{tabular}{|c|c|c|}
\hline $\begin{array}{l}\text { Fatores de } \\
\text { Sucesso }\end{array}$ & $\begin{array}{l}\text { Ambiente } \\
\text { Centralizado }\end{array}$ & Ambiente Distribuído \\
\hline Comunicação & $\begin{array}{l}\text { Mais fácil, devido a proximidade } \\
\text { dos participantes }\end{array}$ & $\begin{array}{l}\text { Crítico pela distância entre participantes. Necessário } \\
\text { Fluxo contínuo }\end{array}$ \\
\hline $\begin{array}{l}\text { Gerenciamento } \\
\text { da equipe }\end{array}$ & $\begin{array}{l}\text { Equipe unida. Facilidade na troca de } \\
\text { informações }\end{array}$ & $\begin{array}{l}\text { Equipe distante. Necessário uma comunicação } \\
\text { padrão }\end{array}$ \\
\hline Credibilidade & $\begin{array}{l}\text { Facilitada pelo melhor conhecimento } \\
\text { da equipe }\end{array}$ & $\begin{array}{l}\text { Deve-se ter confiança e conhecimento da equipe, } \\
\text { mesmo com a distância }\end{array}$ \\
\hline Conflitos & $\begin{array}{l}\text { Pode ocorrer pelo não entendimento } \\
\text { das funções, pouca comunicação, entre } \\
\text { outros. São mais fáceis de resolver }\end{array}$ & $\begin{array}{l}\text { Ocorrem conflitos, porém mais complexos de } \\
\text { resolução, principalmente pelas diferenças culturais } \\
\text { e distância física }\end{array}$ \\
\hline Cooperação & $\begin{array}{l}\text { É necessário cooperação, porém } \\
\text { mais fácil de gerenciar }\end{array}$ & $\begin{array}{l}\text { Exige um grau maior de cooperação, pela distância } \\
\text { da equipe }\end{array}$ \\
\hline $\begin{array}{l}\text { Processos } \\
\text { Definidos }\end{array}$ & Mais fácil de gerenciar & $\begin{array}{l}\text { Ser bem definidos, para melhor coordenação e } \\
\text { entendimento das atividades }\end{array}$ \\
\hline $\begin{array}{l}\text { Diferenças } \\
\text { Culturais }\end{array}$ & Dificilmente presente & $\begin{array}{l}\text { Muito comum. É importante se medir o nível das } \\
\text { diferenças }\end{array}$ \\
\hline
\end{tabular}

A atuação em um ambiente distribuído pode se tornar crítica quando o nível de distribuição física entre os atores é maior e as diferenças culturais são explícitas. Independentemente disto, existe uma forte tendência de empresas distribuírem seus processos, aproveitando incentivos fiscais e buscando vantagens competitivas.

Em PRIKLADNICKI, o autor propõe alguns critérios que definem o nível de distribuição física entre os atores. O primeiro critério é a distância física inter-atores que, considerando os três atores existentes (equipe de desenvolvimento, clientes e usuários), seria a distância física existente entre estes. O segundo critério é a distância física intra-atores, que define a distância física existente dentro de cada equipe de atores.

É difícil encontrar na literatura um material que permita obter critérios e classificações genéricas para ambientes distribuídos. Nesse sentido, muita pesquisa vem sendo feita e muitos autores têm estudado este assunto (SCRIVENER, 1993; LAYZELL, 2000; EVARISTO, 2001), mas o que se pode concluir é que o desenvolvimento distribuído de software é um processo bastante dinâmico que pode ter inúmeras configurações e diversos cenários possíveis, dentro de uma mesma empresa ou apenas dentro de uma mesma área de uma empresa, dependendo do tamanho desta empresa e dos seus objetivos com este tipo de configuração. Mas trabalhar com desenvolvimento distribuído de software é um grande desafio do atual ambiente de negócios, e ter mecanismos capazes de gerir e suportar este tipo de configuração é uma linha de pesquisa que está crescendo cada vez mais.

\section{Protótipo para auxílio do trabalho colaborativo em ambiente fisicamente distribuído}

Devido aos problemas mencionados (principalmente as diferenças culturais e idioma), ocasionados pela distância física entre participantes de um ambiente de desenvolvimento de software, pretende-se, nesta seção, apresentar um protótipo inicial para o auxílio destes participantes situados no ambiente em questão. É importante destacar que não se pretende entrar em detalhes de soluções técnicas que permitam o casamento de ambas tecnologias (CSCW e Desenvolvimento em Ambiente Distribuído), apenas apresentar uma visão geral do que pode vir a se desenvolver numa importante linha de pesquisa. 


\subsection{Protótipo Minotauro}

Batizado de Minotauro, o protótipo tem por objetivo auxiliar o gerenciamento de desenvolvimento de software em ambientes fisicamente distribuídos. A tecnologia utilizada para o desenvolvimento deste protótipo será um ambiente WEB desenvolvido em PHP com o banco de dados PosgreSQL, rodando em um Data Center. A escolha desta tecnologia está fortemente ligada ao fato de serem ferramentas Open Source e de ser o PHP uma linguagem server-side, ocasionando mais segurança para o usuário.

O Minotauro estará disponível em três idiomas (Português, Inglês e Espanhol), sendo possível que o usuário, com apenas um clique, alterne de idioma conforme sua preferência. Porém, os conteúdos inseridos por seus desenvolvedores e gerentes de projetos não serão traduzidos pelo sistema e ficarão no idioma postado.

A figura 1 mostra a tela após o usuário ter efetuado o login no sistema. Esta será a tela principal de visualização para os usuários, sejam eles desenvolvedores ou gerentes de projeto. O Minotauro é composto por três sessões distintas que são as seguintes:

1. Sessão de Menu (subdividida em quatro subseções);

1.1. Projeto: Composto por Informações (Informações relevantes sobre o projeto); Documentos (Documentação do projeto); Prazos (Prazos para execução do projeto); Equipe (Membros que estão envolvidos no projeto).

1.2. Comunicação: Composta pelo Chat (Ferramenta para comunicação on-line com membros do projeto e membros de outros projetos); Fórum (Local para inserir informações de desenvolvimento, novas técnicas a serem compartilhadas com todos os usuários do sistema, participantes ou não de projetos); E-mail (Ferramenta extra para auxilio na comunicação entre os usuários do sistema); Agenda (Informações dos desenvolvedores, gerentes de projeto, telefones úteis e também onde serão programadas datas de entrega de tarefas).

1.3. Coordenação: Composta pelas Tarefas (Item onde o Gerente de Projeto irá atribuir tarefas para sua equipe); Resultados (Gerente de projeto e desenvolvedores poderão ver os resultados obtidos através de gráficos comparativos com tempo de desenvolvimento e aceitação do cliente final de determinada tarefa); Cronograma (Cronograma com datas e prazos para tarefas direcionadas estarem concluídas. Também possibilitará ao Gerente de Projeto uma visão geral de como está o andamento do projeto).

1.4. Colaboração: Composta por Download (Item para serem feitos download de material disponibilizado pelo gerente de projeto ou desenvolvedores); Upload (Item para inserir materiais que serão disponibilizados no item de download); Dicas (Dicas sobre o projeto).

2. Sessão de Conteúdo: Visualização dos dados de itens selecionados (sessão de menu).

3. Sessão de Auxilio ao trabalho cooperativo: Nesta sessão aparecerá os usuário que estão on-line no sistema possibilitando um link rápido para iniciar uma conversa online com o usuário selecionado. Ao lado do nome destes usuários aparecerão bandeiras indicando os idiomas que o usuário domina. Também nessa sessão será possível de ver os projetos que estão em execução e quais são os gerentes destes projetos, sendo possível também interagir com eles em um chat. Ainda nesta sessão 
será possível o usuário escolher outro projeto ao qual ele está trabalhando para se conectar e verificar as opções contidas na sessão de menu.

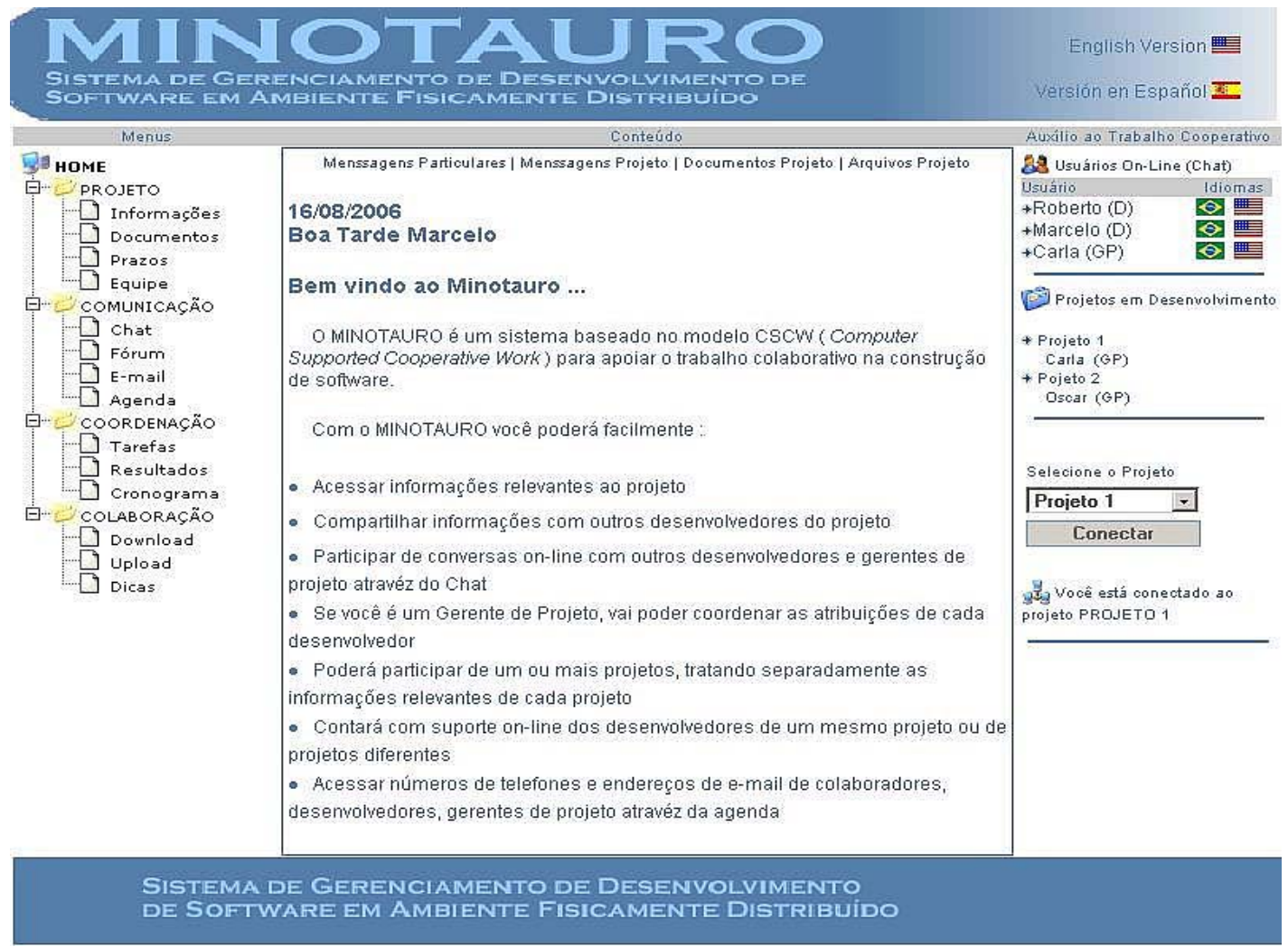

Figura 1 Tela Principal do Minotauro

Com este protótipo poderemos diminuir os problemas relativos à língua, devido a ele ter três opções de idiomas para sua interface, e também será possível uma visão mais ampla do projeto como um todo através de sua sessão de menus. A comunicação, coordenação e colaboração são tratadas de forma específica, compreendendo, assim, uma visão detalhada do projeto em questão. Pretende-se aplicar estudos de caso, em situações de distribuição física, para aperfeiçoamento e validação do protótipo.

\section{Conclusão}

É importante ressaltar algumas características para fins de síntese do presente artigo: as aplicações imediatas para CSCW, apoiadas em groupware, representam um interesse crescente tanto do ponto de vista acadêmico, quanto industrial e comercial; - a vitalidade e a dinâmica internacional, de tendência expansionista, é verificada no uso de groupware, nas organizações modernas; - as flexibilidades oportunizadas, através das inúmeras aplicações possíveis de groupware numa empresa, confirmam os interesses da busca de desenvolvimentos ainda maiores para a área; - as facilidades de comunicação e interação via redes, proporcionaram todos esses avanços, levando os pesquisadores e empresas a novas soluções tecnológicas e organizacionais. As diferenças culturais e suas repercussões negativas em relação a usabilidade dos sistemas CSCW foram analisadas, e se mostrou o grande desafio que elas constituem para os desenvolvedores de sistemas CSCW. 
Por tratar-se de uma pesquisa qualitativa, deve-se ter claro as limitações deste tipo de estudo, principalmente no que se refere ao número de organizações estudadas, restringindo a generalização dos resultados obtidos. Convém destacar que o objetivo da pesquisa foi de contextualizar CSCW e Groupware no Desenvolvimento de Software Fisicamente Distribuído e não implementar um protótipo de ferramenta de auxílio colaborativo, isso pelo fato de não se ter a possibilidade imediata da aplicação do protótipo em situações reais que proporcionam a distribuição física. Desta forma, remete-se esta atividade a trabalhos futuros.

Considera-se este trabalho como uma contribuição relevante para a área de Tecnologia da Informação, visto que busca agregar contribuições de estudos importantes da área da Distribuição Física e Gerência de Projetos de Software no sentido de propor um modelo customizado para auxiliar a colaboração entre os participantes inseridos neste ambiente. Identifica-se um potencial de crescimento nesta linha de pesquisa, onde os pontos fortes envolvem uma parceria estável entre a academia e a indústria, criando condições de experimentação e aprendizagem únicas, decorrentes de uma sinergia positiva entre os parceiros.

\section{Referências}

BOURGES-WALDEGG, L, (1999). "Dealing with Cultural Differences in Computer Supported Co-operative Work". Wokshop on workspace models and collaboration. Department of Comp Science, Queen Mary \& Westfield College, Univ of London, 1999.

EVARISTO, J. R., (2001). “The Management of Distributed Projects Across Cultures”. Submission the Special Issue of IEEE Transactions on Engineering Management, 2001.

FIELDING, R.T., JR., E.J.W., ANDERSON, K.M., BOLCER, G.A., OREIZY, P., TAYLOR, R.N. (1996). "Software engineering and the www: The cobbler's barefoot children, revisited”. Technical report, UCI 96-53, November 1996.

GRUNDY, J.H. MUGRIDGE, R., (1998). "Coordinating distributed software development projects with integrated process modelling and enactment environments". In: Proceedings of IEEE WETICE, Stanford. c1998. p. 39-44.

HERBSLEB, J.D. GRINTER, R.E., (1999). “Splitting the organization and integrating the code: Conway's law revisited”. Proceedings of ICSE, 1999, Los Angeles. IEEECSP.

LAYZELL, P., BRERETON, P., FRENCH, A., (2000). "Supporting Collaboration in Distributed Software Engineering Teams”. Proceedings of the Seventh Asia-Pacific Software Engineering Conference (APSEC.2000).

PRIKLADNICKI, R., (2002). "Problemas, Desafios e Abordagens do Processo de Desenvolvimento de Software”. Trabalho Individual I, Mest. em Computação, PUCRS.

SCRIVENER, S.A.R., HARRIS, D., CLARK, S., ROCKOFF, T., SMYTH, M, (1993). "Designing at a distance via real-time designer-to-designer interaction”. Design Studies, Vol. 14, No.3, pp.261-282.

ZANONI, R; AUDY, J., (2002). "Modelo de Gerencia de Proyectos de Software: Propuesta de Extencion de los Procesos de Gestion do PMI”. In: Anais 3er. Congreso Ibero Americano de Gerencia de Proyectos. Caracas. 